\title{
Gallium transformation under femtosecond laser excitation: Phase coexistence and incomplete melting
}

\author{
O. P. Uteza, ${ }^{1,2}$ E. G. Gamaly,,${ }^{1 *}$ A. V. Rode,${ }^{1}$ M. Samoc, ${ }^{1}$ and B. Luther-Davies ${ }^{1}$ \\ ${ }^{1}$ Laser Physics Centre, Research School of Physical Sciences and Engineering Australian National University, \\ Canberra, ACT 0200 Australia \\ ${ }^{2}$ LP3 - Lasers, Plasmas and Photonic Processes Laboratory, FRE 2165 CNRS - Aix-Marseille II University, Case 917, 163, \\ Avenue de Luminy, 13288 Marseille Cedex 9, France
}

(Received 3 April 2003; revised manuscript received 12 September 2003; published 30 August 2004)

\begin{abstract}
The reversible phase transition induced by femtosecond laser excitation of Gallium has been studied by measuring the dielectric function at $775 \mathrm{~nm}$ with $\sim 200 \mathrm{fs}$ temporal resolution. The real and imaginary parts of the transient dielectric function were calculated using the Fresnel formulae from the absolute reflectivity of a Gallium layer measured at two different angles of incidence. The time-dependent effective electron-phonon collision frequency; the heat conduction coefficient; and the volume fraction of the new phase were recovered directly from the experimental data. The time and space dependent electron and lattice temperatures in the layer undergoing the phase transition were reconstructed without ad hoc assumptions. We converted the temporal dependence of the electron-phonon collision rate into its temperature dependence, and demonstrated that the electron-phonon collision rate has a nonlinear character. This temperature dependence converges to the known equilibrium function during the cooling stage. The maximum fraction of the new phase in the laserexcited Gallium layer reached only $60 \%$ even when the deposited energy was twice the equilibrium enthalpy of melting. We demonstrate that the rate at which the phase transition proceeds and a fraction of the material transformed into the new phase depends strongly on the temperature of the laser-excited Gallium layer, and is restricted by the thickness of this layer which is only several tens of nanometers for the whole range of the pump laser fluences. The kinetics of the phase transformation after the laser excitation can be understood on the basis of the classical theory of the first-order phase transition.
\end{abstract}

DOI: 10.1103/PhysRevB.70.054108 PACS number(s): 78.47.+p, 78.20.Ci, 72.15.Eb, 42.65.Pc

\section{INTRODUCTION}

Phase transitions induced by femtosecond laser pulses in metals and semiconductors are of significant interest for two major reasons: To improve fundamental understanding of the microscopic nature of such phase transitions on the femtosecond time scale and nanometer space scale ${ }^{1-5}$ and to develop possible applications of phase change phenomena in photonics for all-optical switching or optical memories. ${ }^{6-9}$

Over the past twenty years, pump-probe techniques using sub-picosecond laser pulses have been widely used to study laser-induced phase transitions. For example, Kim et al. ${ }^{10}$ measured the transient reflectivity of a laser-excited sample using a single optical probe with time resolution around 100 fs, while Guo et al. ${ }^{11}$ used two optical probes to recover the real and imaginary parts of the full transient dielectric function for a material undergoing a phase transition. The reflectivity measured by optical probes allows changes in the electronic properties of the laser-excited surface to be directly monitored. In order to observe actual structural changes in a lattice on the picosecond time scale both a nonlinear probe ${ }^{12}$ and ultra-short $\mathrm{x}$-ray probes ${ }^{1,13}$ have been employed in addition to optical probes. Several new phenomena have been uncovered by these studies. The general picture that emerges with femtosecond laser excitation is that the electron temperature in a solid rises very rapidly during the pulse while the lattice remains cold. The electrons transfer the absorbed laser energy to the lattice via electron-phonon collisions and heat conduction. Any structural changes that occur in a time shorter than the electron-to-lattice energy transfer time are referred to as nonthermal phase transitions (or, coherent displacement of atoms ${ }^{1}$ ). The phase transition that develops after equilibration of the electron and lattice temperatures is referred to as an ultra-fast thermal phase transition.

A natural question arises, namely, what is the phase state of a solid excited by an ultra-short laser pulse? The numerous reflectivity measurements using a single probe suggest that several picoseconds after the excitation, the solid surface might have completely melted because the maximum reflectivity appears to correspond to that of the molten state. We demonstrate in this paper, however, that the phase transition is, in fact, incomplete as long as $20 \mathrm{ps}$ after the end of the pulse even when the absorbed energy density exceeds twice the equilibrium enthalpy of melting. The experiments are not, however, able to conclusively determine if the resulting transient phase-state is a mixture of crystalline and molten phases or a new, possibly unknown, crystalline phase.

In our previous work we used a single probe to study the time dependent reflectivity of a Gallium film excited by femtosecond laser pulses and observed that the reflectivity increased from $\sim 55 \%$ to $\sim 80 \%$ over a few hundred picoseconds before recovering to its initial value after $\sim 0.1-1 \mu \mathrm{s} .{ }^{7}$ This evolution was attributed to a reversible phase transition from the crystalline $\alpha$-Gallium to the liquid state because the reflectivity maximum was close to that of liquid Gallium. To ensure the process was reversible, the total laser fluence was kept well below the ablation threshold $\left(F_{\text {pump }} \ll F_{\text {abl }}\right)$ and the 
average pulse intensity was below the threshold for plasma formation $\left(I_{\text {laser }} \sim 10^{10} \mathrm{~W} / \mathrm{cm}^{2}\right)$. We have shown from the measurements that the magnitude and rate of the reflectivity changes as well as the recovery time back to the initial state depend on the excitation conditions and background temperature of the Ga film. ${ }^{7}$ However, the transient reflectivity obtained with a single probe beam did not allow us to unambiguously determine the material parameters during the phase transition.

To overcome this deficiency, in this work we replaced the single probe beam by two identical simultaneous 150femtosecond probe beams (at a wavelength of $775 \mathrm{~nm}$ ) set at two different angles of incidence to the sample surface: $12^{\circ}$ and $32^{\circ}$. The simultaneous determination of the transient reflectivity at two angles allowed us to recover the timeresolved real and imaginary parts of the dielectric function $\varepsilon(\omega, t)$ of the Gallium film during the phase transition. From these data it was then possible to calculate the volume fraction of the liquid assuming that the transient state consisted of a mixture of crystalline and liquid Gallium. It was surprising to find that the transformation into the liquid phase never reached $100 \%$ for the whole the observation time (up to 25 ps after the laser pulse). In this paper we argue that the main reason for incomplete phase transformation is the limited depth of heated Gallium layer that extends over only 10 to 20 atomic layers from the surface.

Having measured the transient dielectric function, a number of other important parameters can be directly deduced. Firstly it is possible to calculate the time dependent effective electron-phonon collision frequency (sometimes referred to as optical or transport frequency) as well as the plasma frequency during the phase transition. The transient values of these parameters for the intermediate "mixed" phase created by the laser were found to be drastically different from the equilibrium values for either the crystalline or liquid phases of Ga. Having determined the electron-photon collision frequency from the experimental data, standard relations were used to determine the electron-phonon energy exchange rate which then allowed the space and time dependent electron and lattice temperatures in the laser-excited layer to be reconstructed without any ad hoc assumptions. As a result the dynamics of the phase transformation could be reconstructed both in space and in time. An important conclusion from this analysis is that for a few picoseconds after the pulse, the heat flow from the laser-heated layer has a strongly nonlinear character that evolves to normal linear heat conduction after about 4 ps. Furthermore, throughout the whole interaction time the heated region is restricted to a layer whose thickness is in the range $\approx 30-60 \mathrm{~nm}$ (depending on laser fluence). We point out on the basis of classical theory, that such a thin layer can only support nucleation of the new (molten) phase from seeds whose radius exceeds a critical value that depends strongly on temperature. Using the calculated values of the lattice temperature as a function of fluence, we find that there is a strong correlation between the point that the critical seed radius drops below the thickness of the heated layer and the observed onset of the phase transition. This provides prima facie evidence that the limited thickness of the heated layer plays an important role in suppressing the phase transition even in conditions when the depos-

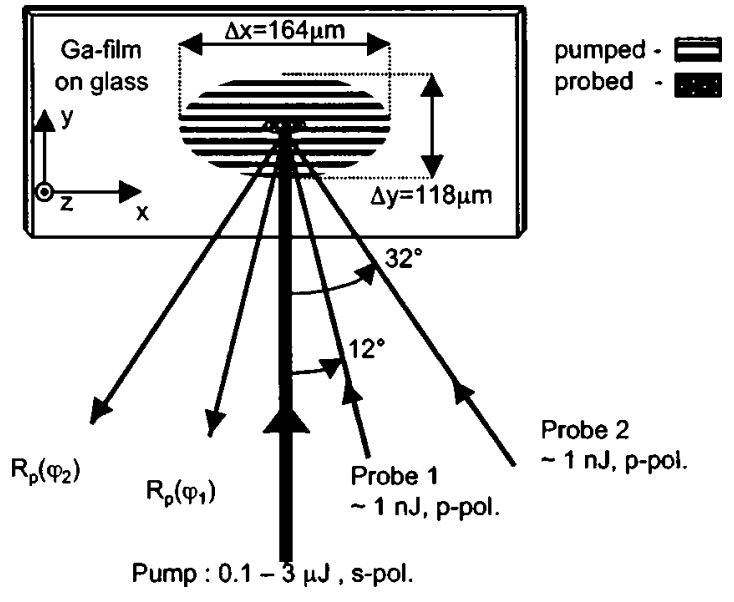

FIG. 1. Arrangement of the pump and two probe beams in the experiments on phase transition induced by femtosecond laser pulses in Gallium films deposited on fused silica substrates.

ited energy significantly exceeds the equilibrium enthalpy of melting.

The paper is organized as follows. The experimental setup, the target structure and diagnostics are described in Sec. II. The experimentally determined transient reflectivity, dielectric function $\varepsilon(t)$, electron-phonon collision rate, plasma frequency, and volume fraction of the new phase are presented in Section III. The heating due to the laser absorption, the electron-phonon energy exchange, and the cooling due to electron heat conduction, all are recovered from the experimental data alone. The threshold energy density for the phase transition and the temperature dependence of the electron-phonon coupling rate are calculated in Sec. IV. The nonequlibrium and thermal stages of phase transformation are considered in a restricted region of the skin layer and discussed in Sec. V, and the conclusions are presented in Sec. VI.

\section{EXPERIMENTAL SETUP}

The experimental arrangement is shown in Fig. 1. A femtosecond Ti:sapphire laser (Clark-MXR CPA 2001, $\lambda$ $=775 \mathrm{~nm}$, pulse duration $t_{p}=150 \mathrm{fs}$, pulse energy $E_{p} \cong 1 \mathrm{~mJ}$, repetition rate $1 \mathrm{kHz}$ ) provided the $s$-polarized pump beam and the two $p$-polarized probes. The target consisted of a 1 to $2 \mu \mathrm{m}$ thick Ga-film deposited on a $1 \mathrm{~mm}$ thick $\mathrm{SiO}_{2}$ substrate. The beams irradiated the target from the silica side with the pump set at normal incidence and the probes at angles of incidence of $12^{\circ}$ and $32^{\circ}$, respectively. The probe beams were tightly focused using $f=100 \mathrm{~mm}$ lenses so that the region probed corresponded to the most uniform central $5 \%$ of the pump spot. The pump and probe beam dimensions, and the beam overlap were monitored using a CCD camera and microscope imaging system. The pump and probe beam areas were $S_{\text {pump }} \approx 6.1 \times 10^{-4} \mathrm{~cm}^{2}$ and $S\left(\phi_{1}\right) \approx S\left(\phi_{2}\right) \approx 2.6$ $\times 10^{-5} \mathrm{~cm}^{2}$. Small probe beams were used to minimize degradation of the temporal resolution caused by the different angles with which the three beams irradiated the target surface. The two probe beams had transverse dimensions of 
$\Delta x\left(\phi_{1}\right)=32 \mu \mathrm{m}, \Delta x\left(\phi_{2}\right)=34 \mu \mathrm{m}$, and this limited the temporal resolution to $\sim 23 \mathrm{fs}$ for probe 1 and $\sim 70 \mathrm{fs}$ for probe 2.

Delay lines place in the optical paths of the pump and probe beams were adjusted to obtain temporal overlap $\left(t_{0}=0\right)$ between the three beams. This was determined by detecting the onset of the pump induced reflectivity change using the probe beams individually while varying the pumpprobe delay time. The resolution of the motion controller used to scan the delay of the pump beam relative to the probes was $\Delta x_{\min }=2 \mu \mathrm{m}$, which corresponds to $\Delta t=13 \mathrm{fs}$, well below the resolution due to the incident angles of the probes. Using $150 \mathrm{fs}$ laser pulses the real temporal resolution was, therefore, limited to about $200 \mathrm{fs}$ in these experiments. The pump and probe beams were cross-polarized (s-polarization for the pump and $p$-polarization for the probes) to minimize the influence of coherent artifacts when the pump and probes overlapped in time, i.e., for time delays within a few hundred fs of $t_{0}=0$.

The pump fluence was varied from 0.1 to $6 \mathrm{~mJ} / \mathrm{cm}^{2}$ using neutral density filters. The probe beam intensities were adjusted to a level at which they induced no detectable changes in the reflectivity. It was also verified that the two probes had no influence on one other. The reflected signals $R_{p}\left(\varphi_{1}=12^{\circ}\right)$ and $R_{p}\left(\varphi_{2}=32^{\circ}\right)$ from the Ga-silica interface were collected by lenses and detected by photodiodes. Iris diaphragms were positioned in the focal planes of the lenses to filter the reflected probe beams and attenuate scattered pump light from the target. The reflectivity of the Ga layer was measured as the ratio of the reflected beam intensity relative to the input beams corrected for the reflection losses from the uncoated silica substrate. The signals from the photodiodes were collected by computer providing the evolution of $R_{p}\left(\varphi_{1}\right)$ and $R_{p}\left(\varphi_{2}\right)$ as a function of the delay between the pump and the two synchronized probe pulses. Each transmitted value was averaged over a sufficient number of pulses (typically 32 laser pulses per delay point) to minimize the influence of pulse-to-pulse fluctuations of the laser. The resulting accuracy of the reflectivity was $\pm 0.5 \%$ at each point. Numerical analysis of the isoreflectance curves for $p$-polarized beams at $12^{\circ}$ and $32^{\circ}$ angles of incidence plotted in the $\{\operatorname{Re}(\varepsilon), \operatorname{Im}(\varepsilon)\}$ plane for both crystalline and liquid Gallium suggested that the resulting accuracy to which the real and imaginary parts of the dielectric functions could be determined was $\pm 2 \%$, and $\pm 1 \%$, respectively. ${ }^{14}$

The Ga-films were deposited using pulsed laser deposition from $6 \mathrm{~N}$-purity $\mathrm{Ga}$ targets onto Silica substrates at $-100^{\circ} \mathrm{C}$ in a chamber pumped to $\sim 2 \times 10^{-6}$ Torr using a Q-switched mode-locked Nd:YAG laser $(\lambda=1.064 \mu \mathrm{m}$, $t_{\mathrm{FWHM}}=60 \mathrm{ps}$, intensity on the target $I_{0} \sim 10^{11} \mathrm{~W} / \mathrm{cm}^{2}$ ) and described in detail elsewhere. ${ }^{6,7}$ The crystalline structure of the deposited Ga-film consisted of the $\alpha$-phase of Gallium that is an elemental metallic molecular crystal containing $\mathrm{Ga}_{2}$ dimers. ${ }^{15-20}$ It has been determined that the covalent bonded $\mathrm{Ga}_{2}$ dimers are oriented almost perpendicular to the film surface. ${ }^{21,22}$ The Ga mirrors were mounted on a Peltier cooler to allow the sample temperature to be varied. The temperature at the rear surface of the Gallium film was maintained at $16^{\circ} \mathrm{C}$ during all the experiments, well below the $\mathrm{Ga}$

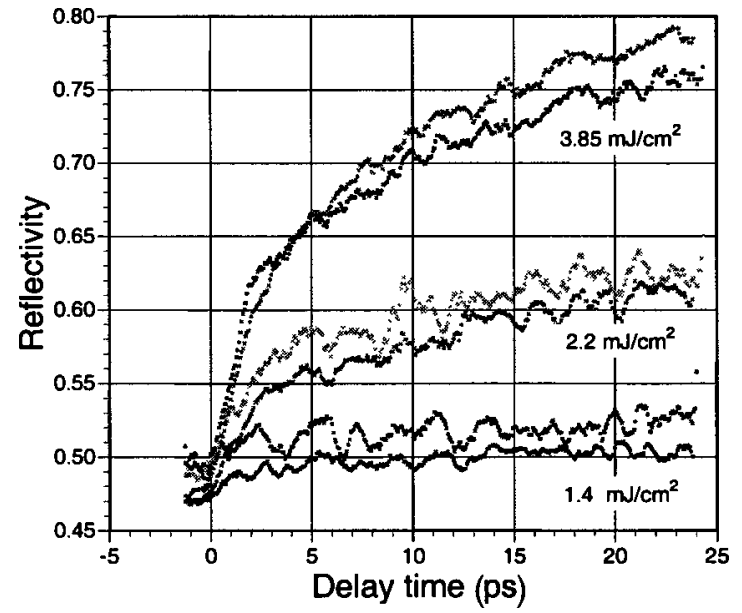

FIG. 2. Probe beams reflectivity change at $12^{\circ}$ and $32^{\circ}$ in the first 25 ps after the pump pulse at $1.4 ; 2.2$; and $3.85 \mathrm{~mJ} / \mathrm{cm}^{2}$. A two-stage reflectivity rise is clearly seen at all laser fluences.

melting point $\left(29.8^{\circ} \mathrm{C}\right)$. The target was translated between each run (at varying pump fluence) to provide a fresh Ga-film zone to minimize any effects of cumulative damage, therefore, ensuring similar initial operating conditions for all the experiments. Auxiliary experiments were performed to check that the fused silica substrate was insensitive to the level of the pump irradiation (i.e., without the Ga-film deposited). No nonreversible changes of the optical properties of the Ga-silica interface were detected for pump fluences up to $F_{\text {pump }} \sim 4 \mathrm{~mJ} / \mathrm{cm}^{2}$ and for $10^{6}$ laser pulses per spot.

\section{EXPERIMENTAL RESULTS}

\section{A. Transient reflectivity}

The reflectivity of the Ga-film, measured at $12^{\circ}$ and $32^{\circ}$ during the first 25 ps after excitation is plotted in Fig. 2 for three pump fluences. It is clear that the reflectivity grows in two-stages: a rapid rise during the first $\sim 2-4$ ps was followed by a slower increase over a much longer period. The reflectivity reached its maximum (not shown on Fig. 2) a few hundred picoseconds after the laser excitation. ${ }^{7}$ This maximum reflectivity exceeded the values at $t=25 \mathrm{ps}$ presented in Fig. 2 by a few percent.

The maximum reflectivity change $\Delta R(\varphi)$ $=R(\varphi, t=+25 \mathrm{ps})-R\left(\varphi_{1}, t=0 \mathrm{ps}\right)$ at and $t=25 \mathrm{ps}$ for a pump fluence $F_{\text {pump }} \sim 3.85 \mathrm{~mJ} / \mathrm{cm}^{2}$ was $\sim 0.3$ for both angles of incidence. The changes in reflectivity induced by $150 \mathrm{fs}$ pulses are consistent with the reflectivity changes measured when the film was heated above its melting point using the Peltier element. The reflectivity changes produced by the Peltier element occur in equilibrium conditions and represent the transition of Gallium from the metallic $\alpha$-crystalline phase to a metal-like liquid state.

The relative reflectivity change at a fixed delay of $25 \mathrm{ps}$ as a function of pump laser fluence is shown in Fig. 3. The threshold fluence leading to an observable change in reflection was $F_{\text {thra }} \sim 0.5-0.7 \mathrm{mJcm}^{-2}$, which is agrees with our previous single-probe experiments. ${ }^{7}$ 


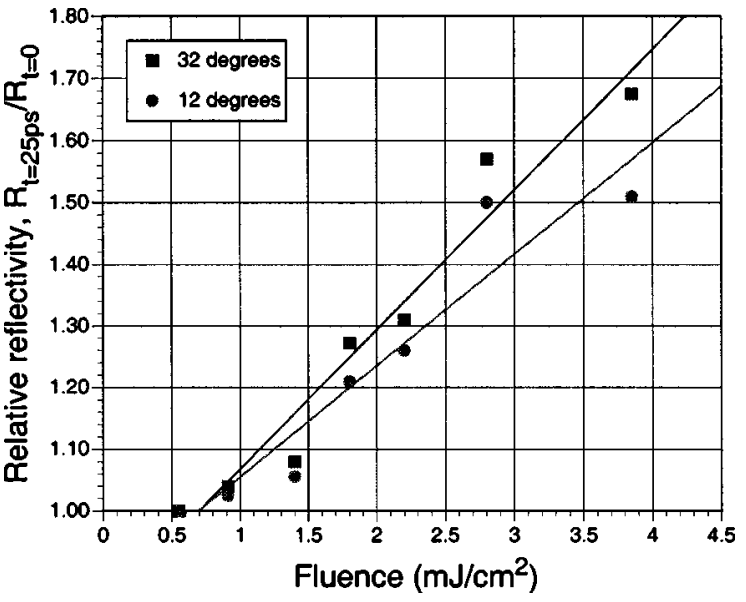

FIG. 3. Relative reflectivity at $25 \mathrm{ps}$ delay time, $R_{p}(t$ $=+25 \mathrm{ps}) / R_{p}(t=0 \mathrm{ps})$, as a function of the pump fluence. Straight lines are linear fits to the data, showing threshold fluence of $0.5-0.7 \mathrm{~mJ} / \mathrm{cm}^{2}$.

For $F_{\text {pump }}>4 \mathrm{~mJ} / \mathrm{cm}^{2}$, the sample was clearly damaged by a few minutes of exposure to the pump pulse at $1 \mathrm{kHz}$ repetition rate $\left(\sim 10^{5}\right.$ laser shots). The damage threshold was significantly lower than the value of $\sim 40-50 \mathrm{~mJ} / \mathrm{cm}^{2}$ measured using a $30 \mathrm{~Hz}$ Ti:sapphire laser. ${ }^{7}$ This difference is probably caused by the higher repetition rate used in the present experiments in combination with the use of the low thermal conductivity fused silica substrate that limits cooling of the Gallium layer between successive laser pulses.

We should note here a consistent feature of all the reflectivity measurements made using either one or two probes, which is the presence of oscillations with a period of about a picosecond, and with an amplitude well above the experimental error or noise. Such oscillations of reflectivity on the picosecond time scale have been observed in a number of experiments $^{23,24}$ and are related to the generation of (1-0.1) THz optical phonons. A detailed study of this phenomenon is out of scope of this paper.

\section{B. Transient dielectric function: Mixture of $\alpha$-Ga and liquid Ga or intermediate transient phase?}

Let us consider as an example, the transient dielectric function determined from the reflectivity measurements [Fig. $4(\mathrm{a})]$ for the intermediate fluence, $F_{\text {pump }} \sim 2.2 \mathrm{~mJ} / \mathrm{cm}^{2}$, that is well below the damage threshold.

The reflection coefficient is related to the real, $\varepsilon_{1}=\operatorname{Re}(\varepsilon)$, and imaginary, $\varepsilon_{2}=\operatorname{Im}(\varepsilon)$, parts of the dielectric function through the Fresnel formulae for either $s$ - and $p$-polarized light. ${ }^{25}$ Thus, two reflectivity measurements at different angles of incidence form a set of two algebraic equations for two unknowns, $\varepsilon_{1}$ and $\varepsilon_{2}$. Numerical solution of these equations as a function of time produces the total complex transient dielectric function of Gallium during the phase transition [see Figs. 4(a) and 4(b)]. For these calculations, we assumed that polarization of probe beams is unchanged by the Ga-film. The a posterior calculated reflectivity values using the dielectric function obtained in accordance to the above procedure is also shown in Fig. 4(a). The solutions accurately reproduced the evolution of the reflectivity observed experimentally. Moreover, good agreement between the calculated dielectric function and that known from the literature for $\mathrm{Ga}$ in the $\alpha$-crystalline and liquid states was obtained when the film was melted using the Peltier element in equilibrium conditions as reported in Table I.

It is clearly seen from Fig. 4(b) that both real and imaginary parts of the dielectric function lie between the values for crystal liquid Gallium (Table I). Thus, the simplest interpretation is that the measured dielectric function, $\varepsilon$, corresponds to that of a mixture of the two equilibrium phases, $\alpha$-Gallium with the dielectric function, $\varepsilon_{a-\mathrm{Ga}}$, and the liquid phase with dielectric function, $\varepsilon_{\mathrm{liq}-\mathrm{Ga}}$.

We, therefore, consider the transient state of Gallium as a homogeneous mixture of two phases ignoring the difference of $0.19 \mathrm{~g} / \mathrm{cm}^{3}$ in the material densities between the crystalline and liquid phases because this represents only $\approx 2 \%$ change. We calculate the volume fraction of liquid using the standard Maxwell Garnett formula, ${ }^{29}$ that gives correct values for limits of both low and high concentrations, and co-
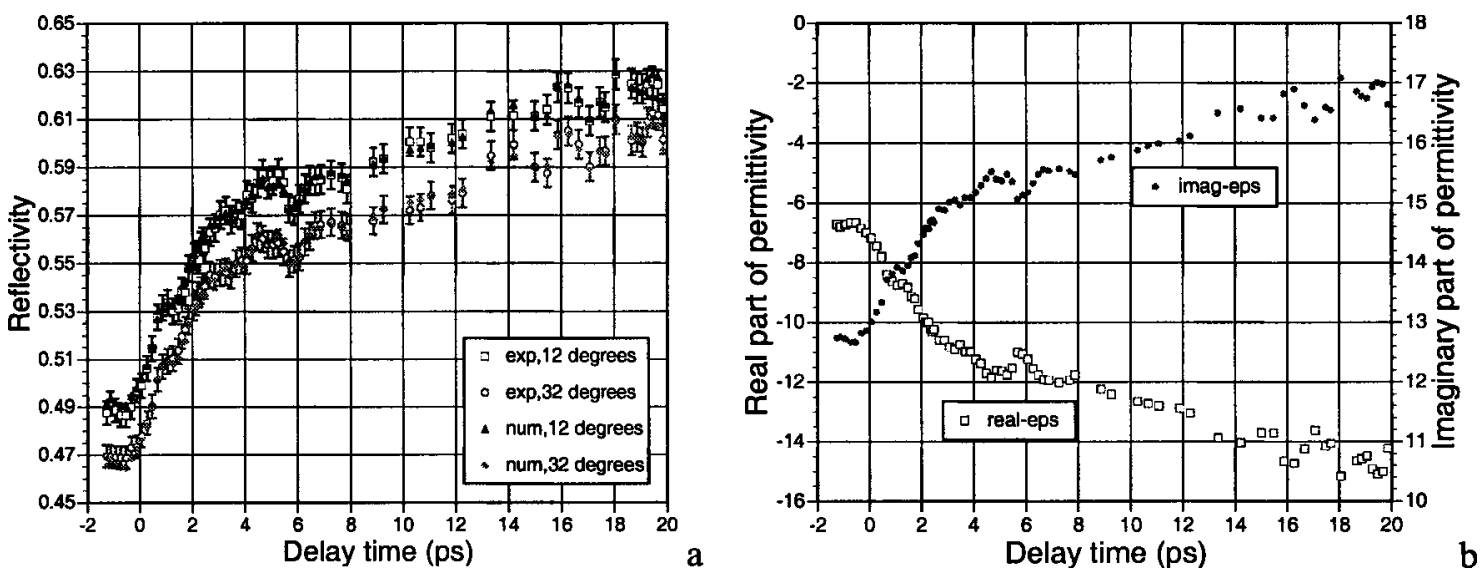

FIG. 4. (a) Measured reflectivity (empty symbols) for $12^{\circ}$ and $32^{\circ}$ at the fluence level $2.2 \mathrm{~mJ} / \mathrm{cm}^{2}$, and numerically restored reflectivity (filled symbols) from the recovered dielectric function by inverting the Fresnel formulae, presented as the calculation's accuracy test; (b) recovered real (empty squares) and imaginary (dots) parts of the dielectric function. 
TABLE I. Dielectric function of Ga, tabulated and recovered from the experiments, for $\alpha$-crystalline and liquid Gallium states at $\lambda=775 \mathrm{~nm}$ (the experimental liquid state values were obtained by melting the film with the Peltier element). The tabulated reference dielectric functions for different Ga states are consistent with the data in Refs. 26-28 and provide independent reflectivity data allowing to check the code accuracy.

\begin{tabular}{lllll}
\hline \hline \multirow{2}{*}{$\varepsilon=\varepsilon_{1}+i \varepsilon_{2}$} & \multicolumn{2}{c}{$\alpha$-crystalline state } & \multicolumn{2}{c}{ Liquid state } \\
\cline { 2 - 5 } & $\varepsilon_{1}$ & $\varepsilon_{2}$ & $\varepsilon_{1}$ & $\varepsilon_{2}$ \\
\hline Tabulated $^{\mathrm{a}}$ & -7.05 & 12.79 & -66.00 & 34.93 \\
Recovered from experiments & -6.85 & 12.82 & -66.89 & 35.36 \\
\hline \hline
\end{tabular}

$\bar{a}$ With courtesy from V. Albanis, University of Southampton, United Kingdom.

incides with an exact Landau formula in the limit of small concentration (up to $40 \%$ ). ${ }^{25}$ Thus, the volume fraction of the liquid phase, $C$, in bulk material formed a crystalline $\alpha$-Gallium determines the dielectric function of the mixture of phases $\varepsilon_{\text {trans }}$ as follows: ${ }^{29}$

$$
\varepsilon_{\text {trans }}=\varepsilon_{\alpha-\mathrm{Ga}}\left[1+\frac{3 C\left(\varepsilon_{\text {liq-Ga }}-\varepsilon_{\alpha-\mathrm{Ga}}\right)}{\varepsilon_{\text {liq-Ga }}+2 \varepsilon_{\alpha-\mathrm{Ga}}-C\left(\varepsilon_{\text {liq-Ga }}-\varepsilon_{\alpha-\mathrm{Ga}}\right)}\right] .
$$

The concentration of liquid Gallium in the transient phase can be calculated by applying this formula to the modulus of the dielectric function $|\varepsilon|=\left(\varepsilon_{1}^{2}+\varepsilon_{2}^{2}\right)^{1 / 2}$ (see Fig. 5). It follows from Fig. 5 that the phase transition into liquid Gallium is not completed during the first $20 \mathrm{ps}$ for the whole range of the pump fluences in these experiments. Furthermore as will be shown later (see Sec. IV B) at the maximum laser fluence of $3.85 \mathrm{~mJ} / \mathrm{cm}^{2}$, the laser energy density deposited into the absorbing layer is more than twice the equilibrium enthalpy of melting of $479.2 \mathrm{~J} / \mathrm{cm}^{3}$. We should note, however, that this interpretation of the physical state of the material as a

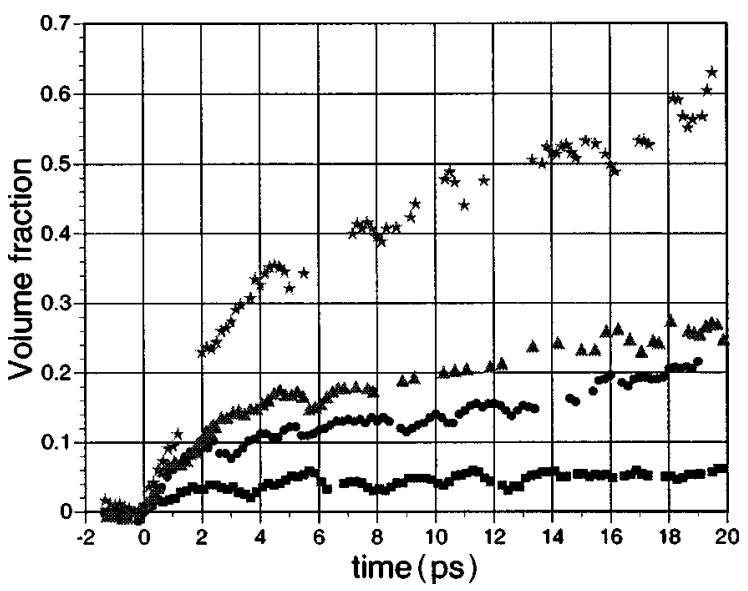

FIG. 5. Volume fraction of the liquid phase in the first $20 \mathrm{ps}$ after the excitation, for the pump fluence $1.4 \mathrm{~mJ} / \mathrm{cm}^{2}$ (squares); $1.85 \mathrm{~mJ} / \mathrm{cm}^{2}$ (circles); $2.2 \mathrm{~mJ} / \mathrm{cm}^{2}$ (triangles); $2.85 \mathrm{~mJ} / \mathrm{cm}^{2}$ (stars), calculated using the recovered from experiments transient dielectric function.
TABLE II. Plasma frequency $\left(\omega_{p} / \omega\right)^{2}$ and electron-phonon optical rate $v_{\text {opt }} / \omega$ for $\alpha$-Ga and liquid Ga for $\lambda=775 \mathrm{~nm}$ at the temperature below and above the melting point; tablulated ${ }^{20,26-28}$ and recovered from the experiments.

\begin{tabular}{lllll}
\hline \hline & \multicolumn{2}{c}{$\left(\omega_{p} / \omega\right)^{2}$} & \multicolumn{2}{c}{$v_{\text {opt }} / \omega$} \\
& Tabulated & Recovered & Tabulated & Recovered \\
\hline \multirow{2}{*}{$\alpha$-Gallium } & 28.4 & 28.8 & 1.59 & 1.63 \\
liquid Gallium & 85.2 & 86.3 & 0.521 & 0.521 \\
\hline \hline
\end{tabular}

mixture of the two limiting phases is only one of the possibilities. We cannot exclude the possibility that the material undergoes progressive transformation into an intermediate phase, which is different from liquid or crystalline Gallium as a result of irradiation.

\section{Electron-phonon efficient collision frequency and plasma frequency in the mixture of phases}

We now use the values of the dielectric function to determine both the electron-phonon collision frequency and the plasma frequency in the heated layer as a function of time. This is possible because $\alpha$-Gallium is a metallic molecular crystal $^{15-18}$ while liquid Gallium ${ }^{19}$ is a free electron-like metal. As a result the dielectric functions of Galluim in equilibrium for either the crystalline or the liquid state are very well described by the Drude-like form: ${ }^{30}$

$$
\varepsilon=1-\frac{\omega_{p}^{2}}{\omega\left(\omega+i v_{\mathrm{opt}}\right)}=1-\frac{\omega_{p}^{2}}{\left(\omega^{2}+v_{\mathrm{opt}}^{2}\right)}+i \frac{\omega_{p}^{2}}{\left(\omega^{2}+v_{\mathrm{opt}}^{2}\right)} \frac{v_{\mathrm{opt}}}{\omega} .
$$

The plasma frequency, $\omega_{p}^{2} / \omega^{2}$, and the effective electronphonon collision frequency (optical rate), $v_{\mathrm{opt}} / \omega$, are both expressed in units of $\omega$, the angular frequency of the probe laser beam $(\omega=2 \pi c / \lambda)$ through the real and imaginary parts of the dielectric function:

$$
\left(\frac{\omega_{p}}{\omega}\right)^{2}=\left(1-\varepsilon_{1}\right)\left[1+\left(\frac{\varepsilon_{2}}{1-\varepsilon_{1}}\right)^{2}\right] ; \frac{v_{\mathrm{opt}}}{\omega}=\frac{\varepsilon_{2}}{1-\varepsilon_{1}} .
$$

This two-parameter form accurately describes the experimentally observed optical properties of both $\alpha$-Gallium, ${ }^{20,26,27}$ and liquid Gallium, ${ }^{26,28}$ in equilibrium conditions at $775 \mathrm{~nm}$ (see Table II). The time-resoled dependence of both $\omega_{p}^{2} / \omega^{2}$ and $v_{\mathrm{opt}} / \omega$ recovered from the reflectivity measurements are presented in Fig. 6.

A close examination of Fig. 6 shows that even at the largest in the figure deposited energy of $2.85 \mathrm{~mJ} / \mathrm{cm}^{2}$, being four times larger than the threshold required to observe any reflectivity changes, neither the electron-phonon collision rate nor the plasma frequency have reached the values for liquid Gallium in equilibrium conditions. The existence of two time stages with different slopes is also evident in Fig. 6; rapid changes occur during the first $2-4 \mathrm{ps}$ followed by a slower evolution over a period of several tens of picoseconds.

For later analysis, it is convenient to approximate the time dependence of both parameters as power laws: 


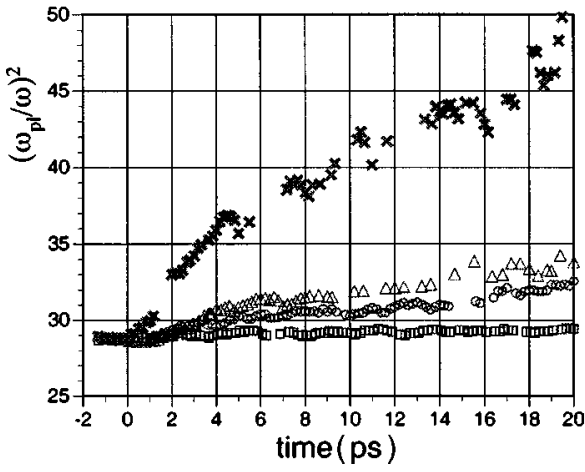

$$
\left(\frac{\omega_{p}}{\omega}\right)^{2} \propto t^{\alpha} ; \frac{v_{\mathrm{opt}}}{\omega} \propto t^{-\beta} ; \alpha, \beta>0 .
$$

The power dependence for the two stages is distinctly different. The initial fast decrease of the electron-phonon collision rate depends strongly on the amount of energy deposited. The interpolation shows that power exponent for the time dependence of the electron-phonon collision rate changes within the limits: $\alpha \sim 0.004-0.124 ; \beta \sim 0.03-0.23$ for the range of fluences used in these experiments. We will use this result in Sec. IV C during discussion of cooling of the surface by electron heat conduction after the pulse.

\section{Relation between the optical rate and the electron-phonon energy exchange rate}

The electron and lattice temperatures bot evolve during the periods associated with absorption of the laser energy; energy transfer from the electrons to the lattice; and lattice cooling by electron heat conduction. The transformation into the new phase and the reverse transition back into the crystalline phase are driven by the electron and lattice temperature distributions both in time and in space. The measured transient real and imaginary parts of the dielectric function have allowed the effective electron-phonon collision rate, $v_{\text {opt }}$ (optical, or transport rate) and the plasma frequency to be calculated. However in a single collision only a small fraction of the energy carried by a heated electron is transferred to the phonon, and hence the electron-to-phonon energy exchange rate, which determines the rate at which the lattice heats up, requires many such collision and occurs much more slower.

The electron-to-phonon energy exchange rate $\nu_{e n}$ can be related to the measured optical rate by the following standard procedure. The conductivity of metals can be conventionally calculated for low values of the adiabaticity parameter $\left(\hbar \omega_{D} / \varepsilon_{F}\right)^{1 / 2} \ll 1$ (at low temperature $T_{L}, T_{e} \ll \varepsilon_{F}$ ), by the means of density functional theory; ${ }^{31}$ here $\omega_{D}$ is the Debye frequency and $\varepsilon_{F}$ is the Fermi energy. In the framework of this theory, the optical (transport) relaxation rate, that defines the conductivity, is related to the phonon frequency averaged by the electron-phonon spectral function as follows:

$$
v_{\mathrm{opt}} \equiv \tau^{-1} \approx 2 \lambda \cdot\langle\omega\rangle ; \omega \gg>\omega_{D}
$$

The $\lambda$-function relates to the effective (re-normalized) electron mass, $m^{*}$. The electron-phonon energy exchange rate
FIG. 6. Plasma frequency $\left(\omega_{p} / \omega\right)^{2}$ and electron-phonon collision rate $v_{\mathrm{opt}} / \omega$ in the first $20 \mathrm{ps}$ after the pump pulse for $\quad 1.4 \mathrm{~mJ} / \mathrm{cm}^{2} \quad$ (squares); $1.8 \mathrm{~mJ} / \mathrm{cm}^{2}$ (circles); $2.2 \mathrm{~mJ} / \mathrm{cm}^{2}$ (triangles), and $2.85 \mathrm{~mJ} / \mathrm{cm}^{2}$ (crosses) laser fluences. (or, the inverse electron-phonon equilibration time $t_{e \text {-ph }}$ ) is given in the two-temperature approximation (electrons and phonons are characterized by separate temperatures, $\left.T_{e}, T_{L}\right)^{32,33}$ in the following form: ${ }^{33}$

$$
v_{\mathrm{en}} \equiv t_{e-\mathrm{ph}}^{-1} \approx \frac{3 \pi \hbar}{2 \varepsilon_{F}} \cdot \lambda \cdot\left\langle\omega^{2}\right\rangle .
$$

We omitted the multiplier that takes into account the weak dependence of the energy exchange rate on the electron temperature ${ }^{33}$ in Eq. (5) because this correction is less than $10 \%$ at its maximum value $\left(T_{e}>T_{L}\right.$ and $\left.T_{e} \ll \varepsilon_{F}\right)$. Now, the relation between the optical and energy exchange rate reads:

$$
\frac{v_{\mathrm{opt}}}{v_{\mathrm{en}}} \approx \frac{4}{3} \frac{\varepsilon_{F}}{\hbar} \frac{\langle\omega\rangle}{\left\langle\omega^{2}\right\rangle} .
$$

The physical meaning of the bracketed frequency is the average phonon frequency and average of the square of the phonon frequency. One can estimate the average phonon frequency in the adiabatic approximation near the Debye temperature $T \sim T_{D}$ as follows: ${ }^{34}$

$$
\langle\omega\rangle \approx\left(\frac{m^{*}}{M}\right)^{1 / 2} \frac{\varepsilon_{F}}{\hbar} ; \quad T_{L} \sim T_{D} .
$$

Thus, the energy exchange rate is linked to the optical rate through the fundamental characteristics of a material as follows: ${ }^{34}$

$$
v_{\mathrm{en}} \approx \frac{3}{4} v_{\mathrm{opt}}\left(\frac{m^{*}}{M}\right)^{1 / 2} .
$$

We adopt this relation for the future calculations. It should be remembered that the time dependence (and therefore, the temperature dependence, as we show below) of the optical rate is obtained directly from the experiments and hence the energy exchange rate can be recovered from the experimental data by applying standard theory. ${ }^{33} \mathrm{We}$ present data for three different metals: Aluminium, ${ }^{43}$ Copper, ${ }^{43}$ and Gallium, ${ }^{20,26-28}$ in equilibrium conditions at $\lambda=775 \mathrm{~nm}\left(\omega=2.43 \times 10^{15} \mathrm{~s}^{-1}\right)$, as examples of these relaxation times in Table III below.

It can be seen from Table III that the energy relaxation time depends strongly on the absorption properties of the material. In highly reflective metals like Copper the relaxation time is of the order of a few picoseconds, while in Gallium it is only $100 \mathrm{fs}$. Note, therefore, that the electronphonon energy exchange time in $\mathrm{Ga}$ is shorter than the 
TABLE III. Optical characteristics of $\alpha$-Ga ( $a$-axis), $\mathrm{Al}$, and $\mathrm{Cu}$ at $775 \mathrm{~nm}$, and calculated optical and electron-phonon energy rates. The $m^{*} / m_{e}$ values are from Ref. 35.

\begin{tabular}{lcccccccc}
\hline \hline & $R$ & $n$ & $k$ & $\varepsilon_{1}$ & $\varepsilon_{2}$ & $m^{*} / m_{e}$ & $t_{\mathrm{opt}}=v_{\mathrm{opt}}^{-1}$ & $t_{e-p h}=v_{\mathrm{en}}^{-1}$ \\
\hline$\alpha-\mathrm{Ga}$ & 0.633 & 2.023 & 3.593 & -8.815 & 14.540 & 1.724 & $0.28 \mathrm{fs}$ & $101 \mathrm{fs}$ \\
$\mathrm{Al}$ & 0.88 & 2.6 & 8.42 & -64.136 & 43.784 & 0.676 & $0.61 \mathrm{fs}$ & $221 \mathrm{fs}$ \\
$\mathrm{Cu}$ & 0.96 & 0.242 & 4.84 & -23.367 & 2.343 & 0.725 & $4.3 \mathrm{fs}$ & $2.29 \mathrm{ps}$ \\
\hline \hline
\end{tabular}

$150 \mathrm{fs}$ laser pulse duration. This indicates, therefore, that for $\alpha$-Ga the lattice temperature will reach its maximum value close to the end of the laser pulse while for copper this will happen a few picoseconds after the end of the pulse.

Taking the known relaxation rates, $v_{\text {opt }}$ and $v_{\text {en }}$, the time history of the electron and lattice temperatures in a solid affected by a short laser pulse can be recovered, and hence the dependence of the effective electron-phonon collision frequency on temperature can be derived. We follow this procedure in Sec. IV below.

Another important parameter, the coefficient of electron heat conduction, $\kappa_{e}$, is also directly related to the measured electron-phonon collision rate:

$$
\kappa_{e}=C_{e}\left(T_{e}\right) n_{e} \frac{l_{e} v_{e}}{3} \approx C_{e}\left(T_{e}\right) n_{e} \frac{v_{F}^{2}}{3 v_{\mathrm{opt}}},
$$

here $C_{e}\left(T_{e}\right)$ is the specific heat for the degenerate electrons, $l_{e}$ is the electron mean free path, $v_{e}$ is the electron speed, and $v_{F}$ is the Fermi velocity $\left(v_{F}=1.92 \times 10^{6} \mathrm{~m} / \mathrm{s}\right.$ for Ga). Therefore, the transient electron and lattice temperatures in the Gallium layer during the laser-target interaction and after the end of the pulse can be calculated without any ad hoc assumptions and only on the basis of the measured reflectivity data.

\section{HEATING AND COOLING PROCESSES}

\section{A. Energy absorption and transient temperature in the Gallium layer}

The laser fluence in all experiments was well below the ablation threshold and, therefore, the density of the target remained essentially unchanged during the interaction, and the laser-matter interaction occurred through the normal skin effect. Since there is no mass or momentum loss, the energy conservation law can be expressed in a two-temperature approximation ( $T_{e}$, for electrons, $T_{L}$, for lattice) by the following set of coupled equations: ${ }^{32,33}$

$$
\begin{gathered}
C_{e} n_{e} \frac{\partial T_{e}}{\partial t}=-\kappa_{e} \nabla T_{e}-n_{e} v_{\mathrm{en}}\left(T_{e}-T_{L}\right)+\nabla \cdot Q_{e}, \\
C_{L} n_{a} \frac{\partial T_{L}}{\partial t}=n_{e} \nu_{\mathrm{en}}\left(T_{e}-T_{L}\right) .
\end{gathered}
$$

Here $C_{e}, C_{L}, n_{e}, n_{a}$, respectively, are the electron and lattice specific heats and the density of free electrons and atoms $\left(n_{a}=5.098 \times 10^{22}\right.$ atom $/ \mathrm{cm}^{3}$ for $\left.\mathrm{Ga}\right) ; Q_{e}$ is the absorbed laser energy per unit time and per unit volume; $v_{\mathrm{en}}$ is the electron- phonon energy exchange rate from Eq. (8), and $k_{e}$ is heat conduction coefficient from Eq. (9). Electron heat conduction dominates in the cooling process, and phonon heat conduction can, therefore, be neglected. The specific heat of the lattice obeys the conventional Dulong-Petit law $C_{L}=3 k_{B}$. The specific heat of the degenerate Fermi electron gas $\left(T_{e} \ll \varepsilon_{F}\right)$ has the conventional form: ${ }^{35}$

$$
C_{e}=\frac{\pi^{2}}{2} \frac{k_{B}^{2} T_{e}}{\varepsilon_{F}}=\frac{\pi^{2} k_{B}^{2} T_{e}}{m^{*} v_{F}^{2}},
$$

where $m^{*}, \varepsilon_{F}$, respectively, are the electron effective mass and the Fermi energy $\left(\varepsilon_{F}=10.4 \mathrm{eV}\right.$ for $\left.\mathrm{Ga}\right) .{ }^{35} Q_{e}$ can be calculated for the normal skin effect interaction mode as: ${ }^{36}$

$$
Q_{e}=\frac{2 A}{l_{s}} I_{0}(r, t) \exp \left(-\frac{2 x}{l_{s}}\right),
$$

here $A$ is the measured absorption coefficient, $l_{s}=c / \omega k$ is the skin depth ( $k$ is the imaginary part of the refractive index, $c$ is the speed of light in vacuum, $x$ is the distance from the surface into the bulk), and $I_{0}=(c / 8 \pi)\left|E_{0}\right|^{2}$ is the incident laser intensity, where $E_{0}$ is the laser electric field.

The characteristic electron heat conduction time $t_{\text {heat }}$, which is defined as the time for the heat wave to propagate a distance equal to the skin depth, can be expressed from Eqs. (9) and (11) as:

$$
t_{\text {heat }}=\frac{3 v_{\mathrm{opt}} l_{s}^{2}}{v_{F}^{2}} .
$$

In case of $\alpha$-Gallium, taking $k=3.64$ for $\lambda=775 \mathrm{~nm},{ }^{20}$ this time $t_{\text {heat }}=3.75 \times 10^{-12} \mathrm{~s}$, which is longer than the pulse duration and the electron-to-lattice energy transfer time estimated above. Therefore, the heating and cooling processes are well separated in time in the experiments.

\section{B. Electron and lattice temperature during the laser pulse}

The transient electron and lattice temperature during the pulse can be calculated in two-temperature approximation [Eq. (10)] using the material parameters recovered from the experiments. The results of the numerical integration of the set of Eq. (10) for a laser pulse with a Gaussian shape in time are presented in Fig. 7 for various laser intensities. The electron temperature rises in the middle of the pulse up to $1000 \mathrm{~K}\left(T_{e} \ll \varepsilon_{F}\right)$ for the highest intensity. The energy transfer from the electrons to the lattice is complete by the end of the laser pulse, $T_{e}=T_{L}=T$ at $t=t_{e-\mathrm{ph}} \cong t_{p}$. As the heating and cooling processes are well separated in time, any cooling processes during the laser pulse can be neglected. Hence, the 


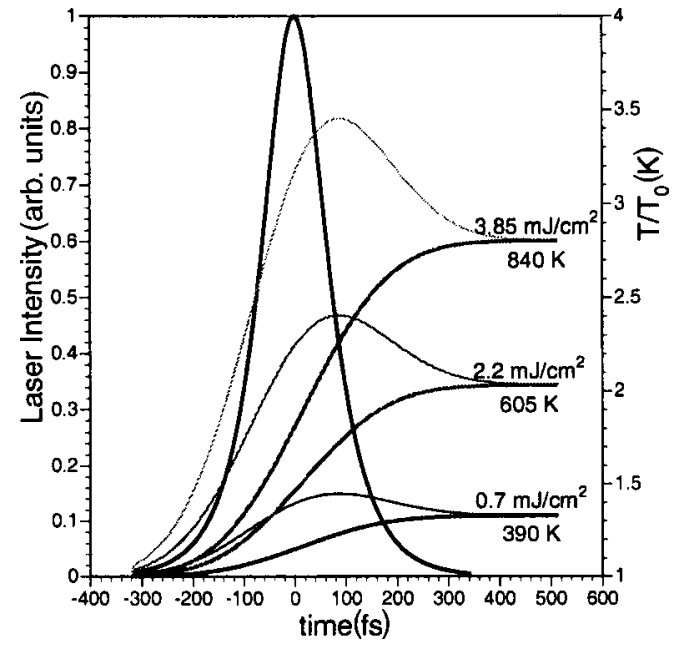

FIG. 7. A Gaussian 150 fs laser pulse, and the electron (thin lines) and lattice (thick lines) temperatures calculated for $0.7 \mathrm{~mJ} / \mathrm{cm}^{2} ; 2.2 \mathrm{~mJ} / \mathrm{cm}^{2}$; and $3.85 \mathrm{~mJ} / \mathrm{cm}^{2}$. The corresponding equilibrated temperature $T_{\max }$ are also shown.

total energy deposited in the Gallium skin layer just after the end of the pulse $\left(t=t_{p}\right), E_{\mathrm{tot}}$, can be estimated as the sum of the absorbed laser energy $Q_{\mathrm{abs}}$ and the internal energy corresponding to the initial constant temperature of the target $Q_{\text {int }}$ $\left(T_{0}=16^{\circ} \mathrm{C}\right.$ or $\left.289 \mathrm{~K}\right)$ :

$$
\begin{gathered}
Q_{\mathrm{abs}}=\left(C_{e} n_{e}+C_{L} n_{a}\right) T\left(t_{p}\right) \approx C_{L} n_{a} T\left(t_{p}\right)=\frac{2 A F}{l_{s}}, \\
C_{L} \gg C_{e}\left(T \ll \varepsilon_{F}\right) ; \quad F=\int_{0}^{t_{p}} I_{0}(t) d t, \\
E_{\mathrm{tot}}=Q_{\mathrm{int}}+Q_{\mathrm{abs}}=C_{L} n_{a} T_{0}+\frac{2 A}{l_{s}} F .
\end{gathered}
$$

The skin depth in $\alpha$-Ga for $775 \mathrm{~nm}$ is $l_{s}=34 \mathrm{~nm}$. Taking the measured absorption coefficient $\mathrm{A} \approx 0.5$, the absorbed energy in the skin layer as a function of the laser fluence can be expressed in the form $E\left[\mathrm{~J} / \mathrm{cm}^{3}\right]=3 \times 10^{5} F\left[\mathrm{~J} / \mathrm{cm}^{2}\right]$. Therefore, the observed threshold fluence for the reflectivity change of $0.7 \mathrm{~mJ} / \mathrm{cm}^{2}$ corresponds to a deposited energy of $210 \mathrm{~J} / \mathrm{cm}^{3}$, which is almost 2.5 times lower than the enthalpy of melting of Gallium in equilibrium conditions, $479.2 \mathrm{~J} / \mathrm{cm}^{3} .41$ The highest fluence in our experiments, $3.85 \mathrm{~mJ} / \mathrm{cm}^{2}$, corresponds to an energy density of $1155 \mathrm{~J} / \mathrm{cm}^{3}$.

The maximum lattice temperature $T_{\max }$ at the silicaGallium interface after equilibration of the electron-lattice temperature can be obtained from Eq. (14):

$$
T_{\text {max }}=T_{0}+\Delta T=T_{0}+\frac{2 A F}{C_{L} n_{a} l_{s}} .
$$

From this we deduce that the reflectivity stars to change $\left(F_{\mathrm{thr}} \cong 0.7 \mathrm{~mJ} / \mathrm{cm}^{2}\right)$ when the maximum lattice temperature reaches $390 \mathrm{~K}$, well above the melting temperature in equilibrium of $303 \mathrm{~K}\left(29.8^{\circ} \mathrm{C}\right)$. When the deposited energy den-

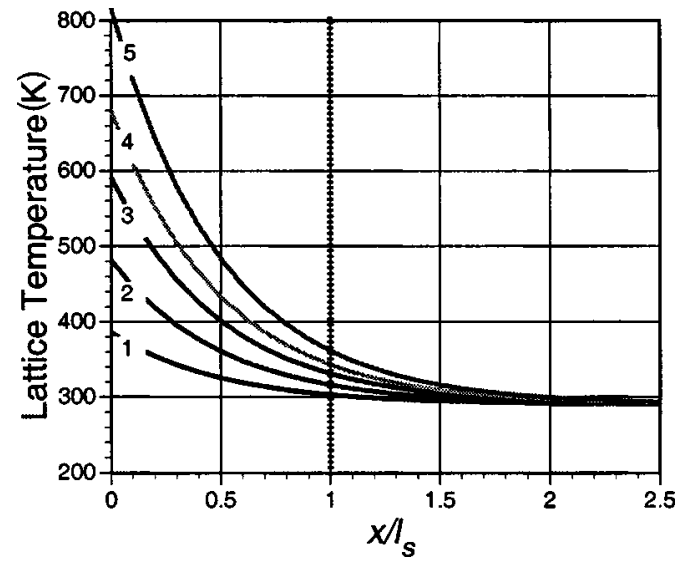

FIG. 8. Maximum lattice temperature distribution with depth in Ga-film at the pump fluence $0.7 \mathrm{~mJ} / \mathrm{cm}^{2}$ (1); $1.4 \mathrm{~mJ} / \mathrm{cm}^{2}$ (2); $2.2 \mathrm{~mJ} / \mathrm{cm}^{2}$ (3); $2.85 \mathrm{~mJ} / \mathrm{cm}^{2}$ (4); $3.85 \mathrm{~mJ} / \mathrm{cm}^{2}$ (5).

sity in the Gallium skin layer exceeds the equilibrium enthalpy of melting, which occurs for fluences above $1.65 \mathrm{~J} / \mathrm{cm}^{2}$, the lattice temperature rises strongly becoming $605 \mathrm{~K}$ at $2.2 \mathrm{~J} / \mathrm{cm}^{2} ; 698 \mathrm{~K}$ at $2.85 \mathrm{~J} / \mathrm{cm}^{2}$; and $840 \mathrm{~K}$ at $3.85 \mathrm{~mJ} / \mathrm{cm}^{2}$. However, the measured reflectivity values in the experiments (for $t<20 \mathrm{ps)} \mathrm{always} \mathrm{remained} \mathrm{slightly}$ lower than those observed for liquid Gallium. Recall that we accurately measured the values for liquid Gallium in equilibrium conditions by heating the sample above it melting temperature to $\sim 309 \mathrm{~K}\left(35^{\circ} \mathrm{C}\right)$ using the Peltier element. As deduced earlier, in the experiments (Figs. 3-6) the phase transition to the liquid state is not completed for at least several tens of picoseconds after the laser pulse in spite of the fact that the electron and lattice temperatures have equilibrated at values far exceeding the melting temperature.

Next consider temperature distribution in the film. In accordance with Eq. (12), the temperature decreases exponentially with distance from the surface:

$$
T=T_{0}+\Delta T_{\max } \exp \left\{-\frac{2 x}{l_{s}}\right\} .
$$

Therefore, the depth of the layer, $0<x<x_{\text {melts, }}$ where the temperature of Gallium exceeds the melting temperature, $T_{\text {melt }}<T<T_{\text {max }}$, can be expressed as follows:

$$
x_{\text {melt }}=\frac{l_{s}}{2} \ln \frac{\Delta T_{\max }}{T_{\text {melt }}-T_{0}} .
$$

Thus, the thickness of the layer, where the phase transition would be expected to occur, is comparable to the skin depth over the whole range of fluences studied (from 0.7 to $3.85 \mathrm{~mJ} / \mathrm{cm}^{2}$ ). It comprises $l_{s}<x_{\text {melt }}<1.8 l_{s}$ $\left(34 \mathrm{~nm}<x_{\text {melt }}<59 \mathrm{~nm}\right)$ which corresponds to only a few tens of atomic layers (see Fig. 8).

\section{Cooling by the electron heat conduction after temperature equilibration}

At $t>t_{e-\mathrm{ph}}$, the energy deposited in the skin-layer is transported away from the layer by means of electron heat con- 
duction in equilibrium conditions, $T_{e}=T_{L}$. The lattice specific heat dominates $\left(C_{L} \gg C_{e}\right)$, while the electrons are responsible for the heat transfer. The set of Eq. (10) reduces to the nonlinear electron heat conduction equations:

$$
\begin{gathered}
\frac{\partial T}{\partial t}=D_{0} \frac{\partial}{\partial x}\left(\frac{T}{T_{\max }}\right)^{1-n} \frac{\partial T}{\partial x}, \\
D=\frac{C_{e} n_{e}}{C_{L} n_{a}} \frac{l_{e} v_{e}}{3}=D_{0}\left(\frac{T}{T_{\max }}\right)^{1-n}, \\
v_{\mathrm{opt}}=v_{0}\left(\frac{T}{T_{0}}\right)^{n} .
\end{gathered}
$$

$T_{\max }$ is the maximum temperature at the moment of electronphonon equilibration from which the lattice cooling starts, $T_{0}$ is the constant temperature in the bulk of the cold Gallium layer that is controlled by the Peltier element. We take the diffusion coefficient, $D_{0}$, as a constant, since the plasma frequency $\left(\omega_{p}^{2} / \omega^{2} \propto n_{e} / m^{*}\right)$ along with the electron effective mass are slowly varying parameters (see Fig. 6). The diffusion coefficient is expressed as follows:

$$
D_{0}=\frac{\pi^{2}}{9} \frac{n_{e}}{n_{a}} \frac{k_{B} T_{\max }}{v_{0} m^{*}}\left(\frac{T_{0}}{T_{\max }}\right)^{n} .
$$

The solution for Eq. (18) is well known. ${ }^{37}$ One can easily obtain the time dependence of the temperature distribution and the propagation of the heat front using the energy conservation law and Eq. (18) as follows:

$$
\begin{gathered}
T(t)=T_{\max }\left(\frac{t_{0}}{t}\right)^{\frac{1}{3-n}}, \\
t_{0}=\frac{l_{s}^{2}}{D_{0}} ; \quad x_{\text {front }}=l_{s} \frac{T_{\max }}{T} .
\end{gathered}
$$

Thus, a nonlinear heat wave with a step-like temperature front propagates inside the bulk of the cold Gallium. The time dependence of the temperature allows us to recover the time dependence of the electron-phonon collision rate:

$$
v_{\mathrm{opt}}=v_{0} T^{n} \propto\left(\frac{t_{0}}{t}\right)^{\frac{n}{3-n}} .
$$

Now, we can relate the experimentally observed time dependence of the electron-phonon collision rate, $v_{\mathrm{opt}} \sim t^{-\beta}$, determined in Sec. III C, to the above formula, to obtain $n$ $=3 \beta /(1+\beta)$. We found earlier that $\beta$ changes in the range from 0.03 to $0.23\left(F=0.7-2.85 \mathrm{~mJ} / \mathrm{cm}^{2}\right)$ during the first 2-4 ps after the heating pulse, and is almost constant throughout the remaining observation time, $4-20 \mathrm{ps}$. That corresponds to changes in $n=0.087-0.56$ in the first $2-4 \mathrm{ps,}$ and $n \approx 1$ after that in the temperature dependence law. Therefore, the heat conduction law depends on the magnitude of the laser fluence and changes with time after the pulse in the following manner: When $n \approx 0$ heat conduction is very nonlinear with $T \sim t^{-1 / 3}$, while when $n \rightarrow 1$ it tends to the conventional process, $T \sim t^{-1 / 2}$.
The effective electron-phonon collision rate in liquid Gallium in equilibrium conditions is proportional to temperature, $v_{\mathrm{opt}} \sim T / T_{D}$. The experimental measurements in the temperature range up to $860 \mathrm{~K}$ are in close agreement with the linear law. ${ }^{26,28}$ Hence, the temperature dependence of the electron-phonon collision rate tends to that for liquid $\mathrm{Ga}$ in equilibrium conditions as the laser intensity (fluence) increases. The resulting expression for the recovered nonlinear dependence of the electron-phonon collision rate on temperature, $v_{\text {opt }} \sim v_{0} T^{n}(n<1)$, is another confirmation of the fact that the crystal-to-liquid phase transition was incomplete for the whole range of laser intensities studied here.

The characteristic cooling time, $t_{0}$, [see Eq. (20)] corresponds to the time for the heat wave to propagate over a distance equal to $l_{s}$. It is inversely proportional to the deposited energy density:

$$
t_{0}=\frac{l_{s}^{2}}{D_{0}} \propto T_{\max }^{1-n},
$$

here $T_{\max }$ is given by Eq. (15). This time is much longer than the electron-phonon equilibration time, therefore, $n \sim 0$. Taking $n_{e}=3 n_{a}, m^{*}=m_{e}, v_{0}=2 \times 10^{15} \mathrm{~s}^{-1}$ for the mixture of phases, and $T_{0}=289 \mathrm{~K}$, one obtains $t_{0}[s]=4.6$ $\times 10^{-8} / T_{\max }[\mathrm{K}]$. Thus, the characteristic cooling time ranges from $120 \mathrm{ps}\left(0.7 \mathrm{~mJ} / \mathrm{cm}^{2}\right)$ to $55 \mathrm{ps}\left(3.85 \mathrm{~mJ} / \mathrm{cm}^{2}\right)$. One can also obtain the time for the temperature in Gallium layer to decrease back to the melting point of $T_{\text {melt }}=303 \mathrm{~K}: t_{\text {melt }}$ $=t_{0}\left(T_{\text {max }} / T_{\text {melt }}\right)^{2}$, ranging from $0.23 \mathrm{~ns}$ for $0.7 \mathrm{~mJ} / \mathrm{cm}^{2}$ to $0.43 \mathrm{~ns}\left(3.85 \mathrm{~mJ} / \mathrm{cm}^{2}\right)$. We consider that as the time when the reverse liquid-to-crystal phase transition takes place. This time is in a good agreement with our previous observation with a single probe beam. ${ }^{7}$

\section{DISCUSSION}

The change in the material properties induced by the femtosecond laser pulses occurs in a different way during two periods. The first period comprises the time interval shorter than the electron-to-lattice energy transfer time, $t<t_{e \text {-ph }}$. The absorbed energy is deposited into the electrons but the lattice remains cold. The material transition during this time interval is referred to as a "nonthermal - nonequilibrium" phase transition. During the second period, at $t>t_{e-\mathrm{ph}}$, the transition proceeds in conditions for which the electron and lattice temperatures are equilibrated. In this case the phase transition is considered as a thermal one.

The material transformation takes place in a thin layer that comprises only several tens of atomic layers and the thickness of the layer heated above the phase transition temperature varies rapidly in time. We consider, therefore, the implications of the space and time constraints on the kinetics of the phase transformation in such conditions.

\section{A. The nonequilibrium stage, $T_{e} \gg T_{L}$}

During the nonequilibrium stage that lasts $\sim 100 \mathrm{fs}$ the electrons gain an average energy in excess of the melting temperature. Any excited electron produces a change in the inter-atomic potential $\Delta U \sim T_{e}$. This change in turn results in 
atomic oscillations $\delta R$ around the initial equilibrium position (i.e., the generation of optical phonons):

$$
\Delta U \sim\left(\frac{\partial^{2} U}{\partial R^{2}}\right) \delta R^{2} \sim \frac{J_{i}}{d^{2}} \delta R^{2},
$$

here $d$ and $J_{i}$ are the inter-atomic distance and the ionization potential, respectively. The average amplitude of atomic oscillation can then be estimated as follows: ${ }^{34,38}$

$$
\delta R=d\left(\frac{T_{e}}{J_{i}}\right)^{1 / 2} .
$$

According to Ref. 38, the average displacement of an atom for the temperature range of $400-1000 \mathrm{~K}$, typical for melting of the majority of solids, is $0.2-0.25$ of the elementary cell radius. The atomic displacement following from Eq. (24) is of the order of a few tenths of an Angstrom. The period of these oscillations is of the same order of magnitude as the time necessary to shift an atom into a position corresponding to a new phase. It can be estimated in the framework of the adiabatic approximation. ${ }^{34}$ The momentum of an oscillating atom can be estimated from $p \delta R \sim \hbar$. The Virial theorem provides the relation between the oscillation and the kinetic energy $M \omega^{2} \delta R^{2} \sim p^{2} / 2 M$. The oscillation period thus could be estimated as follows:

$$
t \sim \frac{M \delta R^{2}}{\hbar} .
$$

This time for Gallium equals to approximately 100 femtoseconds and agrees with similar calculations for $\mathrm{Si}$ and GaAs. ${ }^{4}$ The process described above can be considered as a "coherent displacement" of lattice layers in the heated skin layer due to electron excitation. In other words, this is a fast (on the time scale of the laser pulse) loss of long-range (crystalline) order on the space scale of the whole laser-excited layer. There are two other forces that can also lead to coherent displacement of the lattice during the time before electron-phonon equilibration: The gradient of the electronic pressure, and the ponderomotive force of the laser electric field in the skin layer. The effect of coherent displacement manifests itself in nonlinear phenomena such as secondharmonic generation (involving loss of symmetry), ${ }^{12}$ or in the change of the x-ray diffraction intensity. ${ }^{1}$ In both of the studies reported in Refs. 1 and 12 the electron-phonon energy exchange time was several ps and hence significantly longer than the laser pulse duration ( $\sim 100 \mathrm{fs})$. Therefore, the effect of nonthermal modification was pronounced.

In the experiment described in this paper, the time for loss-of-long-range-order due to a coherent displacement is comparable to the laser pulse duration and to the electron-tophonon energy exchange time. Therefore, any nonthermal phase transition could not be observed because of the limited $200 \mathrm{fs}$ resolution in the experiments.

\section{B. Phase transitions during the thermal state, $T_{e}=T_{\text {Lattice }}$}

A crystal lattice heated above its phase transition temperature passes into an unstable state ${ }^{39}$ with thermal oscillations of the atoms at elevated temperature allowing them to shift to positions close to that of the new molten phase. The transformation into the new phase is, therefore, seeded by this unstable state.

There are two processes that can drive the thermal phase transition for a heated Gallium skin layer confined between a cold glass substrate and the unheated Gallium that remains at a constant temperature below the melting point. The first possibility is that the phase transition is surface assisted. Since the maximum temperature occurs near the SilicaGallium interface this suggests that transformation into the new phase would be energetically favored at this interface provided the surface tension between the liquid Gallium and silica is lower than that between the silica and crystal-liquid Gallium interface. ${ }^{40}$ However, the surface tension between the liquid Gallium and silica is unknown to the best of our knowledge and hence it is difficult to assess the likelihood of this process.

Alternatively, as shown in the standard text by Landau and Lifshitz, ${ }^{39}$ the formation of liquid "seeds" within an internally heated bulk crystal with its boundaries held at constant temperature below the melting point is energetically favoured for bulk melting. In our experiments the thickness of the overheated layer increases with fluence increasing the volume in which seeds may form. Consequently, we consider below the implications for melting of the formation of seeds of liquid Ga within the heated Gallium layer.

Small seeds of the new molten phase are created in the overheated layer due to lattice fluctuations. ${ }^{39}$ These seeds are, however, unstable structures because the formation of an interface between the two phases requires extra energy to overcome the surface tension at that interface. Hence seeds with a size less than a critical value will decay back into the initial phase, while seeds with a size exceeding this critical radius will grow rapidly driving the transformation of the bulk into the new molten phase. The critical radius of a seed is related to the temperature of the overheated layer, $T$, as follows: ${ }^{39}$

$$
r_{\mathrm{cr}}=\frac{2 \alpha}{P^{\prime}-P} \approx \frac{2 \alpha}{n_{a}\left(T-T_{\mathrm{melt}}\right)} .
$$

Here $\alpha$ is the surface tension between the crystal and liquid Gallium $(\alpha=720$ dyne $/ \mathrm{cm}),{ }^{40} P^{\prime}$ is the transient pressure in the skin layer, and $P$ is the pressure corresponding to the melting temperature, $P=n_{a} T_{\text {melt }}$. We have neglected the small difference in the densities of liquid and solid $\mathrm{Ga}$ $\left[\rho_{\text {solid }}=5.907 \mathrm{~g} / \mathrm{cm}^{3} ; \rho_{\text {liquid }}=6.095 \mathrm{~g} / \mathrm{cm}^{3}\right.$ near and above the melting point at $\left.29.8^{\circ} \mathrm{C}(\operatorname{Ref} 41)\right]$ in the above calculations. A clear consequence of Eq. (26) is that the critical radius increases rapidly as the temperature drops towards the melting temperature. As a result when the thickness of the heated layer is very small, as is the case in these experiments, the critical seed radius may exceed the layer thickness strongly inhibiting seed formation and slowing the rate at which the phase transition occurs. 
Landau and Lifshitz also showed that the probability of a seed forming is an exponential function of the critical radius: ${ }^{39}$

$$
w \propto \exp \left(-\frac{4 \pi \alpha r_{\mathrm{cr}}^{2}}{3 T}\right)
$$

This probability drops very rapidly with increasing radius. Hence two factors affect a change of phase seeded by fluctuations within the thin laser heated layer: Firstly if the average temperature in the layer is too low, the critical seed radius exceeds the layer thickness and this inhibits the phase transition; and secondly if the average layer temperature is low, the probability of a seed forming is exponentially small.

The time dependence of the lattice temperature, discussed above, must also be taken into account in this picture. The lattice temperature grows very rapidly during the pulse (Fig. 7) and afterwards decreases more slowly due to heat losses from the skin layer. Hence the critical radius reaches it smallest value near the end of the pulse and then steadily increases during the cooling phase. At the same time the probability of forming seeds peaks at the end of the pulse and then drops much more rapidly because of its strong sensitivity to temperature $\left[\approx \exp \left(-8 \pi \alpha^{2} / 3 \mathrm{~T}^{3}\right)\right.$ for $\left.T \gg T_{\text {melt }}\right]$. Hence there is only a very short period during which the phase transition can actually occur and this is probably the major reason why we observe only a partial change of phase in all condition of these experiments in spite of the fact that sufficient energy has been fed into the surface layer and its temperature far exceeds that for equilibrium melting.

Using Eq. (26) and using the average temperature in the heated layer which can be deduced from Fig. 8, we can calculate that the minimum seed radius decreases from $>70$ to $15 \mathrm{~nm}$ as the fluence increases from 1.5 to $2.85 \mathrm{~mJ} / \mathrm{cm}^{2}$ (Fig. 9). It is clear that the phase transformation at $F<1.5 \mathrm{~mJ} / \mathrm{cm}^{2}$ is strongly inhibitied even if temperature exceeds that for melting because the radius of a critical seed is larger than the thickness of the layer with $T>T_{\text {melt }}$. It follows from Eq. (26), that the critical radius coincides with the skin depth when the lattice temperature is still $270 \mathrm{~K}$ above the melting temperature.

Finally it is also worth noting that during femtosecond excitation of any material, the pressure inside the skin layer increases with the temperature. In the Gallium skin layer, the pressure varies from 3 to $5 \mathrm{kbar}$ for fluences in the range $0.7 \mathrm{~mJ} / \mathrm{cm}^{2}<F<2.85 \mathrm{~mJ} / \mathrm{cm}^{2}$. The melting temperature of $\mathrm{Ga}(\mathrm{I})$ decreases with increasing pressure: At $1.2 \mathrm{GPa}(12 \mathrm{kbar})$ the melting temperature has dropped to $293 \mathrm{~K}$ (room temperature). ${ }^{42}$ These changes could affect the dynamics of the phase transition although the change in melting temperature is small compared to the overheating induced by the laser in the interaction region.

\section{CONCLUSION}

In this paper, the reversible phase transition in a Gallium film irradiated by femtosecond laser pulses has been studied by measuring its transient reflectivity with one pump and two identical simultaneous femtosecond probes set at two differ-

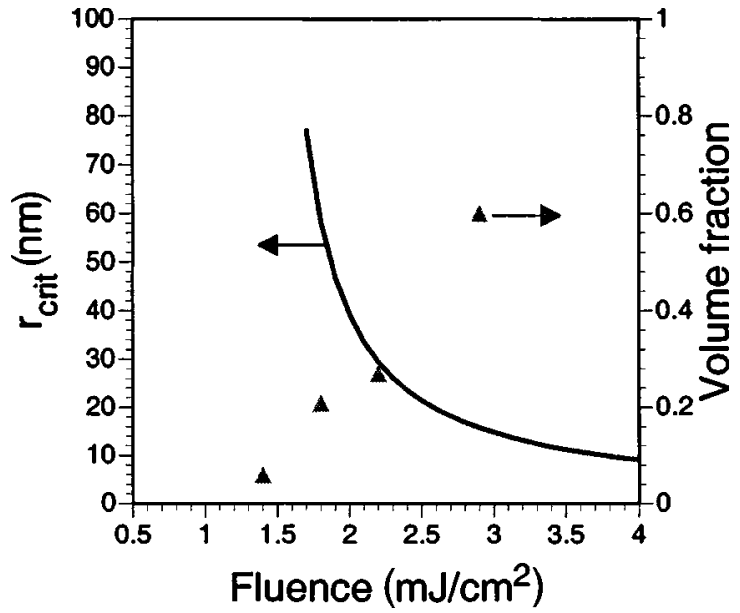

FIG. 9. The calculated critical radius of seeds and volume fraction of a new phase recovered from the experiments vs pump fluence.

ent angles. These measurements allowed complete determination of the real and imaginary parts of the transient dielectric function with $\sim 200$ fs time resolution and from these the electron-phonon effective collision rate (optical or transport frequency) and heat conduction coefficient for the first $25 \mathrm{ps}$ after irradiation. The time-dependent electron and lattice temperatures in the layer undergoing the phase transition were then determined. The time history of the phase transition in Gallium induced by femtosecond laser pulses was reconstructed on the basis of the experimentally measured transient effective electron-phonon collision frequency in the first $25 \mathrm{ps}$ after the excitation with $\sim 200$ fs time resolution.

The main results of this study are the following:

- Under femtosecond excitation, $\alpha$-Ga transforms into a phase state whose optical properties are intermediate between those for the crystal and for the liquid Gallium. This state is a most likely a coexistence of different phases of Gallium, possibly $\alpha$-Gallium and liquid Gallium.

- A likely reason for the incomplete phase transition is the limited thickness of the laser-excited layer. Near the energy threshold for the reflectivity change the critical size of a seed needed for growth of the new phase is comparable to or greater than the skin depth. This strongly inhibits the phase tranformation making it drastically different from the phase transition in a bulk solid in the conditions of thermodynamic equilibrium.

- The experimentally determined threshold for the reflectivity changes, $0.5-0.7 \mathrm{~mJ} / \mathrm{cm}^{2}$, corresponds to an absorbed energy density two times lower than the equilibrium enthalpy of melting for Gallium.

- The electron-phonon collision rate and the heat conduction coefficients recovered from the experimental data are transient nonlinear functions of temperature, and are drastically different from those for equilibrium melting.

- The first sharp rise in reflectivity in a few ps after the excitation corresponds to the fast growth of the new phase during which electron heat conduction is negligible. The following slow increase in reflectivity corresponds to the conditions when the heat transfer dominates. The lattice cooling through the electron heat conduction slows down, and then 
terminates the solid-to-liquid phase transformation on $100-200$ ps timescale. $^{7}$

- The measured changes in the linear (specular) reflection occurs on the timescale of several picoseconds or greater. The phase transition therefore proceeds in the thermal mode.

The nonthermal stage of the phase transition or, the coherent displacement, takes place during the time comparable to the $150 \mathrm{fs}$ pump duration and could not be resolved in these experiments with $200 \mathrm{fs}$ resolution presented here. The coherent displacement of the lattice excited by the laser pulse on a time-scale shorter than the electron-phonon energy exchange time $(<100 \mathrm{fs})$, can be studied in future experiments combining ultra-short optical and $\mathrm{x}$-ray probing ${ }^{1}$ with time resolution better than $100 \mathrm{fs}$. These experiments would lead to a precise characterization of the nonequilibrium stage of the laser-excited phase transition.

\section{ACKNOWLEDGMENTS}

The authors are grateful to N. I. Zheludev for a number of useful discussions and suggestions at the early stage of the experiments, and to V. Albanis for the set of data on optical properties of Gallium. O.U. gratefully acknowledges Australian Research Council's (ARC) financial support through the ARC-IREX scheme (Grant No. X00106527 "Ultrafast laser ablation and deposition of thin films"). The work has been partly supported by other ARC schemes.
*Author to whom correspondence should be addressed. Electronic mail: gam110@rsphysse.anu.edu.au

${ }^{1}$ A. Rousse, G. Rischel, S. Fourneaux, I. Uschmann, S. Sebban, G. Grillon, Ph. Balcou, E. Förster, J. P. Geindre, P. Audebert, J. C. Gauthier, and D. Hulin, Nature (London) 410, 65 (2001).

${ }^{2}$ A. Cavalleri, K. Sokolowski-Tinten, J. Bialkowski, and D. von der Linde, Appl. Phys. Lett. 72, 2385 (1998).

${ }^{3}$ K. Sokolowski-Tinten, J. Bialkowski, M. Boing, A. Cavalleri, and D. von der Linde, Phys. Rev. B 58, R11 805 (1998).

${ }^{4}$ P. Stampfli and K. H. Bennemann, Phys. Rev. B 49, 7299 (1994).

${ }^{5}$ K. Seibert, G. C. Cho, W. Kütt, H. Kurz, D. H. Reitze, J. I. Dadap, H. Ahn, M. C. Downer, and A. M. Malvezzi, Phys. Rev. B 42, 2842 (1990).

${ }^{6}$ V. Albanis, S. Dhanjal, K. MacDonald, P. Petropoulos, H. L. Offerhaus, D. J. Richardson, A. V. Rode, and N. I. Zheludev, J. Lumin. 87-89, 646 (2000).

${ }^{7}$ A. V. Rode, M. Samoc, B. Luther-Davies, E. G. Gamaly, K. F. MacDonald, and N. I. Zheludev, Opt. Lett. 26 (7), 441 (2001).

${ }^{8}$ P. J. Bennett, S. Dhanjal, P. Petropoulos, D. J. Richardson, N. I. Zheludev, and V. I. Emelianov, Appl. Phys. Lett. 73, 1787 (1998).

${ }^{9}$ P. Petropoulos, H. S. Kim, D. J. Richardson, V. A. Fedotov, and N. I. Zheludev, Phys. Rev. B 64, 193312 (2001).

${ }^{10}$ A. M.-T. Kim, J. P. Callan, C. A. D. Roester, and E. Mazur, Phys. Rev. B 66, 245203 (2002).

${ }^{11}$ C. Guo, G. Rodriguez, A. Lobad, and A. J. Taylor, Phys. Rev. Lett. 84, 4493 (2000).

${ }^{12}$ S. V. Govorkov, I. L. Shumai, W. Rudolf, and T. Shroder, JETP Lett. 52, 117 (1990).

${ }^{13}$ C. W. Siders, A. Cavalleri, K. Sokolowski-Tinten, Cs. Toth, T. Guo, M. Kammler, M. Horn von Hoegen, K. R. Wilson, D. von der Linde, and C. P. J. Barty, Science 286, 1340 (1999).

${ }^{14}$ W. R. Hunter, Appl. Opt. 21, 2103 (1982); C. A. D. Roeser, A. M.-T. Kim, J. P. Callan, L. Huang, E. N. Glezer, Y. Siegal, and E. Mazur, Rev. Sci. Instrum. 74, 3413 (2003).

${ }^{15}$ X. G. Gong, G. L. Chiarotti, M. Parrinello, and E. Tosatti, Phys. Rev. B 43, 14277 (1991).

${ }^{16}$ X. G. Gong, G. L. Chiarotti, M. Parrinello, and E. Tosatti, Europhys. Lett. 21 (4), 469 (1993).

${ }^{17}$ O. Züger and U. Dürig, Phys. Rev. B 46, 7319 (1992).

${ }^{18}$ M. Bernasconi, G. L. Chiarotti, and E. Tossatti, Phys. Rev. B 52,
9988 (1995).

${ }^{19}$ O. Hunderi and R. Ryberg, J. Phys. F: Met. Phys. 4, 2096 (1974).

${ }^{20}$ R. Trittibach, Ch. Grutter, and J. H. Bilgram, Phys. Rev. B 50, 2529 (1994).

${ }^{21}$ W. J. Huisman, J. F. Peters, M. J. Zwanenburg, S. A. de Vries, T. E. Derry, D. Abernathy, and J. F. van der Veen, Nature (London) 390, 379 (1997).

${ }^{22}$ D. A. Walko, I. K. Robinson, Ch. Grütter, and J. H. Bilgram, Phys. Rev. Lett. 81, 626 (1998).

${ }^{23}$ M. Hase, K. Ishioka, M. Kitajima, S. Hishita, and K. Ushida, Appl. Surf. Sci. 197-198, 710 (2002).

${ }^{24}$ A. M. Lindenberg, I. Kang, S. L. Johnson, T. Missalla, P. A. Heimann, Z. Chang, J. Larsson, P. H. Bucksbaum, H. C. Kapteyn, H. A. Padmore, R. W. Lee, J. S. Wark, and R. W. Falcone, Phys. Rev. Lett. 84, 111 (2000).

${ }^{25}$ L. D. Landau and E. M. Lifshitz, Electrodynamics of Continuous Media (Pergamon Press, Oxford, 1984), p. 293.

${ }^{26}$ R. Sh. Teshev and A. A. Shebzukhov, Opt. Spectrosc. 65, 693 (1988).

${ }^{27}$ R. Kofman, P. Cheyssac, and J. Richard, Phys. Rev. B 16, 5216 (1977).

${ }^{28}$ N. R. Comins, Philos. Mag. 25, 817 (1972).

${ }^{29}$ J. C. M. Garnett, Philos. Trans. R. Soc. London, Ser. A 203, 385 (1904).

${ }^{30}$ E. M. Lifshitz and L. P. Pitaevskii, Physical Kinetics (Pergamon Press, Oxford, 1981).

${ }^{31}$ E. G. Maksimov, D. Yu. Savrasov, and S. Yu. Savrasov, Phys. Usp. 40, 337 (1997).

${ }^{32}$ M. I. Kaganov, I. M. Lifshitz, and L. V. Tanatarov, Sov. Phys. JETP 4, 173 (1957).

${ }^{33}$ P. B. Allen, Phys. Rev. Lett. 59, 1460 (1987).

${ }^{34}$ Yu. A. Il'inskii and L. V. Keldysh, Electromagnetic Response of Material Media (Plenum Press, New York, 1994).

${ }^{35}$ C. Kittel, Introduction to Solid State Physics (Wiley, New York, 1996).

${ }^{36}$ E. G. Gamaly, A. V. Rode, and V. T. Tikhonchuk, Phys. Plasmas 9, 949 (2002)

${ }^{37}$ Ya. B. Zel'dovich and Yu. P. Raizer, Physics of Shock Waves and High-Temperature Hydrodynamic Phenomena (Academic Press, New York, 1967). 
${ }^{38}$ J. M. Ziman, Principles of the Theory of Solids (University Press, Cambridge, 1964).

${ }^{39}$ L. D. Landau and E. M. Lifshitz, Statistical Physics (Pergamon Press, Oxford, 1980).

${ }^{40}$ G. Kaptay, E. Báder, and L. Bolyán, Mater. Sci. Forum 329-330, 151 (2000).
${ }^{41}$ CRC Handbook of Chemistry and Physics, 60th ed., edited by R. C. Weast and M. J. Astle (CRC Press, Boca Raton, 1981).

${ }^{42}$ T. Kenichi, K. Kazuaki, and A. Masao, Phys. Rev. B 58, 2482 (1998).

${ }^{43}$ Handbook of Optics, edited by M. Bass (McGraw Hill, New York, 1995), Vol. II. 